\title{
Multiaxial fatigue criteria applied to motor crankshaft in thermoelectric power plants
}

\author{
Marcos Venicius S. Pereira ${ }^{1}$, Fathi Aref Darwish $^{2}$, André Feiferis ${ }^{1}$ and Tiago Lima Castro ${ }^{1.1}$ \\ ${ }^{1}$ Department of Chemical and Materials Engineering, Catholic University of Rio de Janeiro, Rua \\ Marquês de São Vicente, 225, Rio de Janeiro, 22451-900, RJ, Brazil \\ ${ }^{2}$ Department of Civil Engineering, Fluminense Federal University, Rua Passo da Pátria, 156, Niterói, \\ 24210-240, RJ, Brazil
}

\begin{abstract}
Fatigue failures of motor crankshafts operating in thermoelectric power plants have recently been reported. Stress fields provided by finite element calculations at critical points of a crankshaft that failed in service are used to test the structural integrity of the component. Taking into account the fact that the stresses acting at a given point are most likely out of phase, multiaxial fatigue criteria based on the von Mises stress are considered to be most suitable for predicting the fatigue behavior of the crankshaft. Using the von Mises stress, it was also possible to apply octahedral shear stress-based criteria and the results obtained have indicated that the crankshaft made of DIN 34CrNiMo6 steel should not suffer fatigue failure under the action of the stress fields in question. However, such failures have been occurring and this apparent discrepancy is presented and briefly discussed in the present study.
\end{abstract}

\section{Introduction}

Historically, the evaluation of the fatigue behavior of metallic materials was based on the determination of uniaxial fatigue test parameters for life prediction. However, many mechanical components such as railroad wheels, crankshafts, axles and turbine blades are expected to experience time-varying multiaxial stresses during their in-service lifetime. Accordingly, the need has been arising to introduce multiaxial high cycle fatigue damage criteria capable of predicting fatigue failure under such loading conditions.

The present work deals with the case of a motor crankshaft which suffered fatigue failure during operation in a power generating thermoelectric plant. The crankshaft is presented schematically in Fig. 1, showing its main journals and indicating the sequence of firing, adopted so as to insure uniform rotational motion of the crankshaft. Stresses and strains developed in the crankshaft during rotation were estimated numerically via finite element method using a commercial Ansys software. Twenty critical locations, exhibiting high stress levels, were seen to exist at the root of the junctions between the journals and their corresponding webs. As one may expect, these stresses are elastic in nature and hence they can be used to predict the possibility of fatigue failure by applying stress-based high

Corresponding author: tiagocastrobl@gmail.com 
cycle fatigue criteria [1-7] at critical locations, where fatigue damage is capable of initiating. Although the stress components acting at a given point are synchronous, they are generally out of phase and hence applying a failure criterion using individual stress amplitudes and mean stress values is seen to be a difficult task. However, in addition to providing the variation of each stress component during a full cycle of the crankshaft, the finite element calculations also provide the three principle stresses as well as the corresponding von Mises stress, incorporating whatever phase difference existing between the individual components of the stress tensor. Accordingly, the fatigue failure criteria to be applied in the present work are those based on static yield criteria models. More specifically, Mises, Sines and Crossland models are reviewed and then applied to the stress fields acting at twenty critical locations of the crankshaft.

\section{Reviewing the models}

\subsection{Mises equivalent stress criterion}

Knowing the maximum and minimum values of von Mises stress at a given point, both the corresponding amplitude and mean value can be respectively expressed as

$$
\begin{gathered}
\sigma_{m i s, a}=\frac{\Delta \sigma_{m i s}}{2}=\frac{\sigma_{m i s, m a x}-\sigma_{m i s, m i n}}{2} \\
\sigma_{m i s, m}=\frac{\sigma_{m i s, \text { max }}+\sigma_{m i s, m i n}}{2}
\end{gathered}
$$

and the criterion for fatigue resistance is given by [8]

$$
\frac{\Delta \sigma_{m i s}}{2} \leq f_{-1}^{\prime}
$$

where $f_{-1}^{\prime}$ is the fatigue resistance limit in the presence of a mean stress $\sigma_{m i s, m}$.

Based on the Goodman diagram, $f_{-1}^{\prime}$ can be related to the fatigue limit stress in fully reversed bending $f_{-1}$ by the expression [9]

$$
f_{-1}^{\prime}=f_{-1}\left[1-\frac{\Delta \sigma_{m i s, m}}{\sigma_{u}}\right]
$$

where is the ultimate tensile strength.

On the other hand, if one adopts the Gerber relation, expressed in terms of, will have the form [9]

$$
f_{-1}^{\prime}=f_{-1}\left[1-\left(\frac{\Delta \sigma_{m i s, m}}{\sigma_{u}}\right)^{2}\right]
$$




\subsection{Sine's criterion}

Here the octahedral shear stress is proposed to be used as a fatigue damage criterion. The physical significance of the octahedral shear stress is that it expresses the average effects of slippage on different planes and in different directions of all crystals in the aggregate, with slip in any given grain caused by the critical resolved shear stress in that grain [10]. As static torsion does not influence either cyclic bending or cyclic torsion fatigue limits and as static tension and compression linearly influence the fatigue limit in both tension and torsion, Sines' criterion considers that the mean hydrostatic stress during a cycle has an effect on fatigue life. The resulting failure criterion can thus be expressed as [8]

$$
\frac{\Delta \tau_{o c t}}{2}+\alpha_{s}\left(3 \sigma_{h}\right) \leq \beta_{s}
$$

where $\Delta \tau_{\text {oct }}$ is the octahedral shear stress range, $\sigma_{h}$ is the mean hydrostatic stress during a cycle and $\alpha_{s}$ and $\beta_{s}$ are constants that can be determined by applying specific cyclic loading conditions. It is important to point out that Sines criterion pertains to the static yield-based models group given the relationship between $\Delta \tau_{\text {oct }}$ and $\Delta \sigma_{\text {mis }}$ shown below [8].

$$
\Delta \tau_{\text {oct }}=\frac{\sqrt{2}}{3} \Delta \sigma_{m i s}
$$

The determination of the constants $\alpha_{s}$ and $\beta_{s}$ can be achieved by considering two different cyclic loading conditions. For example: a fully reversed uniaxial cyclic normal stress (stress ratio $R=-1$ ), with the fatigue resistance limit $f_{-1}$, and another loading condition corresponding to repeated bending $(R=0)$, with the fatigue resistance limit $f_{0}$ related to $f_{-1}$ by the Goodman relation [9] according to the following expression:

$$
f_{0}=\frac{f_{-1}}{1+\frac{f_{-1}}{\sigma_{u}}}
$$

With these loading conditions, $\alpha_{s}$ and $\beta_{s}$ can be calculated as:

$$
\begin{gathered}
\beta_{s}=\frac{\sqrt{2}}{3} f_{-1} \\
\alpha_{s}=\frac{\sqrt{2}}{3}\left(\frac{f_{-1}-f_{0}}{f_{0}}\right)
\end{gathered}
$$

and hence the criterion is finally expressed as

$$
\frac{\Delta \tau_{\text {oct }}}{2}+\frac{\sqrt{2}}{3}\left(\frac{f_{-1}-f_{0}}{f_{0}}\right)\left(3 \sigma_{h}\right) \leq \frac{\sqrt{2}}{3} f_{-1}
$$

Now for fully reversed torsion, $3 \sigma_{h}$ is nil and $\Delta \tau_{\text {oct }} / 2$ is equal to $\sqrt{2} t_{-1} / \sqrt{3}$, meaning that $\beta_{s}$ will be given by $\sqrt{2} t_{-1} / \sqrt{3}$. For $\beta_{s}$ to be unique, $t_{-1}$ has to be equivalent to 
$f_{-1} / \sqrt{3}$, which is seen to be a serious limitation on the Sines criterion as it disagrees with experimental results where $t_{-1} / f_{-1}$ varies from 0.5 for mild metals to 1 for brittle metals $[1,2]$.

Another form of the Sines criterion can be presented as [1]

$$
\sqrt{J_{2, a}}+\kappa\left(\sigma_{h}\right)=\lambda
$$

where $I_{2, a}$ is the amplitude of the second invariant of the deviatoric component of the stress tensor. Here $\kappa$ and $\lambda$ are constants to be determined by applying two different cyclic loading conditions as, for example, fully reversed torsion and repeated bending $(R=0)$, thus yielding the constants $\kappa$ and $\lambda$ :

$$
\begin{gathered}
\kappa=\frac{3 t_{-1}}{f_{0}}-\sqrt{3} \\
\lambda=t_{-1}
\end{gathered}
$$

and the criterion will be represented by

$$
\sqrt{J_{2, a}}+\left(\frac{3 t_{-1}}{f_{0}}-\sqrt{3}\right)\left(\sigma_{h}\right)=t_{-1}
$$

It is important to point out that equations (11) and (15) are equivalent, which can simply be demonstrated by replacing $t_{-1}$ in equation (15) by $f_{-1} / \sqrt{3}$ and multiplying the two sides by $\sqrt{2} / \sqrt{3}$ thus yielding the following relation.

\subsection{Crossland's criterion}

Here the mean hydrostatic stress is replaced by the maximum hydrostatic stress during a loading cycle and the criterion is thus represented by [11]

$$
\frac{\Delta \tau_{o c t}}{2}+\alpha_{c}\left(3 \sigma_{h, \max }\right) \leq \beta_{c}
$$

For $R=-1 ; \quad 3 \sigma_{h, \max }=f_{-1}$

For $R=0 ; \quad 3 \sigma_{h, \max }=f_{0}$

Accordingly,

$$
\beta_{c}=\frac{\sqrt{2}}{3} f_{-1}\left(\frac{f_{0}}{2 f_{0}-f_{-1}}\right)
$$

and

$$
\alpha_{c}=\frac{\sqrt{2}}{3}\left(\frac{f_{-1}-f_{0}}{2 f_{0}-f_{-1}}\right)
$$




\section{Applying the models}

This can be achieved by substituting the stress components acting at a given point of the crankshaft in the left-hand side (LHS) of the inequalities corresponding to the models in question. However, such components are expected to be most likely out of phase and hence the application of static yield criteria models is best achieved on the basis of von Mises stress along a cycle of the crankshaft, thus $\Delta \tau_{\text {oct }}$ can be determined using equation (7).

A comparison between the two sides, for each of the inequalities given by expressions (3), (11) and (17), would indicate whether fatigue failure would or not occur. With the right-hand side (RHS) considerably exceeding the left-hand side (LHS), one may conclude that the structural integrity of the component in question would be maintained for "infinite" fatigue life. Such a comparison can be formalized by considering a fatigue resistance index $I$, defined by

$$
I=\frac{L H S-R H S}{R H S}
$$

\section{Results and discussion}

The maximum and minimum values of the von Mises stress, estimated numerically via FEM at twenty critical points along a full cycle of the crankshaft, are presented in Table 1. The steel's fatigue properties needed to apply the models, namely $f_{-1}, t_{-1}, f_{0}$ and $\sigma_{u}$, are presented in Table 2, together with the constants $\beta_{s}, \alpha_{s}, \beta_{c}$ and $\alpha_{c}$, calculated respectively, according to equations (9), (10), (18) and (19).

Based on expressions (3), (11) and (17), one can compare the two sides for each of the three inequalities and therefore calculate the fatigue resistance index $I$, for the loading conditions in question. The individual $I$ values obtained at the twenty critical locations of the crankshaft are given in Fig. 2, for the Mises, Sines and Crossland criteria. A comparison of the average $I$ values corresponding to the three criteria is presented in Fig. 3. The difference between Mises criterion, on the one hand, and Sines and Crossland criteria, on the other hand, is attributed to the fact that the latter two models consider that the hydrostatic stress component affects the fatigue behavior of the material.

As a result of applying the three models in question, one verifies that the driving force for fatigue fracture, represented by the cyclic stresses, is invariably inferior to the fatigue resistance limit, defined by the RHS of the inequality, meaning that fatigue failure is not liable to take place. This does not correspond to the reality of the situation where failure of the crankshaft did in fact occur. Largely negative values of $I$, which are indicative of no failure, could be due to an overestimated RHS and/or underestimated LHS of the inequalities involved in the criteria.

An underestimated LHS could be caused by adopting high safety factor and/or could be related to a possible underestimation of the stress fields provided by the finite element calculations. Another important factor leading to such a discrepancy may ultimately be related to microstructural aspects of the steel. In fact, preliminary metallographic investigation has revealed the presence of large elongated nonmetallic inclusions capable of reducing the fatigue resistance limit down to a level considerably lower than that actually taken into account. 
Table 1. Maximum and minimum levels of von Mises stress, together with its range, amplitude and mean value, at twenty critical locations of the crankshaft.

\begin{tabular}{|c|c|c|c|c|c|c|}
\hline $\begin{array}{c}\text { Crackpin } \\
\text { Journal }\end{array}$ & $\begin{array}{c}\text { Connecting } \\
\text { Rod }\end{array}$ & $\begin{array}{c}\sigma_{\text {mis, max }} \\
{[\mathrm{MPa}]}\end{array}$ & $\begin{array}{l}\sigma_{\text {mis, } \min } \\
\text { [ MPa ] }\end{array}$ & $\begin{array}{c}\Delta \sigma_{\text {mis }} \\
{[\mathrm{MPa}]}\end{array}$ & $\begin{array}{c}\sigma_{\mathrm{mis}, \mathrm{m}} \\
{[\mathrm{MPa}]}\end{array}$ & $\begin{array}{c}\sigma_{\text {mis, a }} \\
\text { [ MPa ] }\end{array}$ \\
\hline \multirow{2}{*}{1} & A & 155 & -38 & 193 & 58 & 96 \\
\hline & $B$ & 199 & -208 & 408 & -4 & 204 \\
\hline \multirow{2}{*}{2} & A & 221 & -228 & 450 & -3 & 225 \\
\hline & B & 183 & -182 & 366 & 0 & 183 \\
\hline \multirow{2}{*}{3} & A & 186 & -160 & 347 & 12 & 173 \\
\hline & B & 206 & -199 & 405 & 3 & 202 \\
\hline \multirow{2}{*}{4} & A & 177 & -175 & 352 & 0 & 176 \\
\hline & $B$ & 211 & -228 & 440 & -8 & 220 \\
\hline \multirow{2}{*}{5} & A & 209 & -216 & 425 & -3 & 212 \\
\hline & B & 147 & -92 & 239 & 27 & 119 \\
\hline \multirow{2}{*}{6} & A & 142 & -144 & 287 & -0 & 143 \\
\hline & B & 195 & -41 & 237 & 77 & 118 \\
\hline \multirow{2}{*}{7} & A & 166 & -44 & 211 & 61 & 105 \\
\hline & $B$ & 141 & -55 & 197 & 42 & 98 \\
\hline \multirow{2}{*}{8} & A & 144 & -54 & 199 & 45 & 99 \\
\hline & B & 120 & -59 & 179 & 30 & 89 \\
\hline \multirow{2}{*}{9} & A & 108 & -74 & 182 & 17 & 91 \\
\hline & B & 127 & -68 & 195 & 29 & 97 \\
\hline \multirow{2}{*}{10} & A & 121 & -82 & 204 & 19 & 102 \\
\hline & B & 171 & -91 & 262 & 40 & 131 \\
\hline
\end{tabular}

Table 2. Fatigue properties of the DIN34CrNiMo6 steel, together with the values of $\alpha_{s}, \alpha_{c}, \beta_{s}$ and $\beta_{c}$.

\begin{tabular}{|c|c|c|c|c|c|c|c|}
\hline $\begin{array}{c}f_{-1} \\
{[\mathrm{MPa}]}\end{array}$ & $\begin{array}{c}t_{-1} \\
{[\mathrm{MPa}]}\end{array}$ & $\begin{array}{c}\sigma_{u} \\
{[\mathrm{MPa}]}\end{array}$ & $\begin{array}{c}f_{0} \\
{[\mathrm{MPa}]}\end{array}$ & $\alpha_{s}$ & $\alpha_{c}$ & $\begin{array}{c}\beta_{s} \\
{[\mathrm{MPa}]}\end{array}$ & $\begin{array}{c}\beta_{c} \\
{[\mathrm{MPa}]}\end{array}$ \\
\hline 414 & 239 & 1035 & 296 & 0.188 & 0.312 & 195 & 324 \\
\hline
\end{tabular}




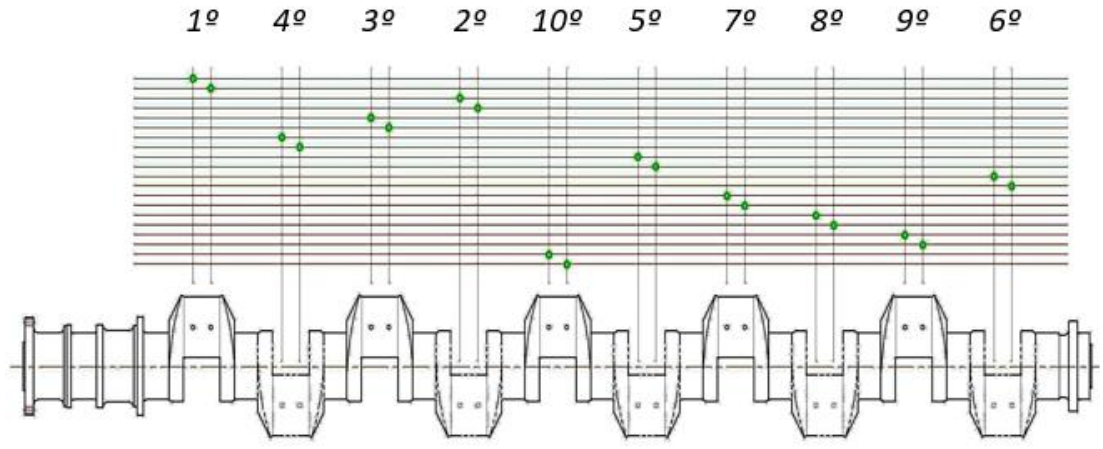

Fig. 1. Schematic of the crankshaft, showing its main journals and indicating the sequence of firing.

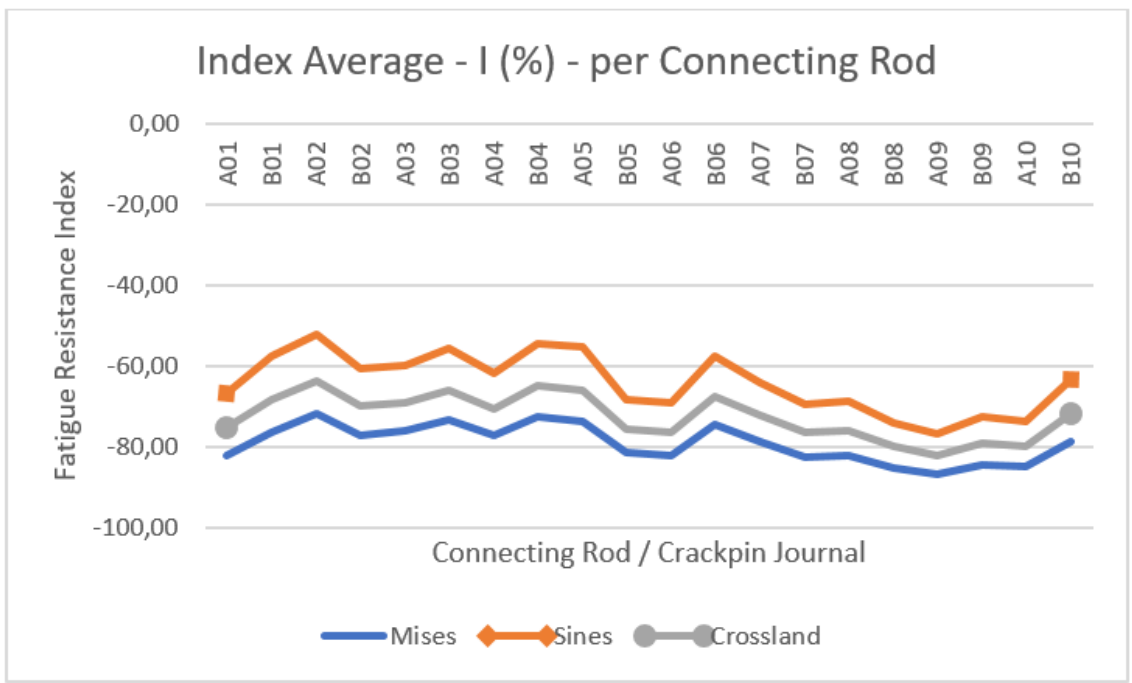

Fig. 2. Fatigue Resistance Index, as determined at twenty different locations of the crankshaft.

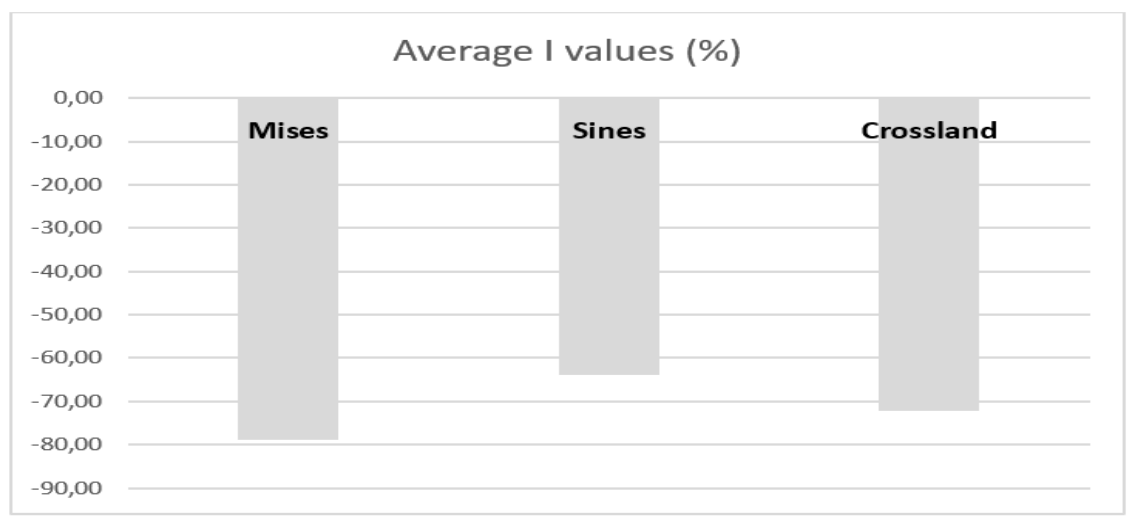

Fig. 3. Average values of the fatigue resistance index for the three models in question. 


\section{Conclusion}

Based on what is presented above, the following conclusion can be drawn:

Applying the Mises, Sines and Crossland models, it is verified that the driving force for fatigue failure represented by the stress fields acting at critical points of the crankshaft is lower than the fatigue resistance limit of the steel. The occurrence of fatigue failure, despite such a verification, can be rationalized on the basis of a possible underestimation of the stress fields provided by the finite element calculations and / or an overestimation of the fatigue resistance limit which is a function of microstructural aspects of the steel.

\section{References}

1. I.V. Papadopoulos, P. Davoli, C. Gorla, M. Filippini, A. Bernasconi. A comparative study of multiaxial high cycle fatigue criteria for metals. Int J Fatigue, 19: 219-35 (1997)

2. A. Carpinteri, A. Spagnoli. Multiaxial high cycle fatigue criterion for hard metals, Int J Fatigue, 23: 135-45 (2001)

3. A. Carpinteri, A. Spagnoli, S. Vantadori. Multiaxial fatigue assessment using a simplified critical plane based criterion. Int J Fatigue, 33: 969-76 (2011)

4. A. Carpinteri, A. Spagnoli, S. Vantadori. C. Bagni. Structural integrity assessment of metallic components under multiaxial fatigue: the C-S criterion and its evolution. Fatigue Fract Eng Mater Struct, 36: 870-83 (2013).

5. A. Carpinteri, M. Kurek, T. Lagoda, S. Vantadori. Estimation of fatigue life under multiaxial loading by varying the critical plane orientation. Int J Fatigue, 100: 512-20 (2017)

6. A. Fatemi, N. Shamsaei. Multiaxial fatigue: An overview and some approximation models for life estimation. Int J Fatigue, 33: 948-58 (2011).

7. Y. Liu, S. Mahadevan. Multiaxial high cycle fatigue criterion and life prediction for metals. Int J Fatigue, 27: 790-800 (2005).

8. D. F. Socie, G. B. Marquis, Multiaxial fatigue, Society of Automotive Engineers SAE, Warrendale USA (2000).

9. J. Schijve, Fatigue of Structures and Materials, Springer, Delft, The Netherlands (2009).

10. G. Sines. Behavior of metals under complex static and alternating stresses. In: Metal Fatigue, McGraw-Hill, New York, p. 145-69 (1959).

11. B. Crossland. Effect of large hydrostatic pressures on the torsional fatigue strength of an alloy steel. In: Proceedings of the international conference on fatigue of metals. Institution of Mechanical Engineers, London, p. 138-49 (1956). 